\title{
Hilbert Modular Surfaces and the Classification of Algebraic Surfaces
}

\author{
F. Hirzebruch (Bonn) and A. Van de Ven (Leiden)
}

Herrn K. Stein zum 60. Geburtstag gewidmet

\section{Introduction}

Around 1900 Hilbert, Hecke ([7]), Blumenthal ([2]) and others started the study of certain 2-dimensional complex spaces, which are closely related to the classification of special types of 2-dimensional abelian varieties. These complex spaces can easily be described. In fact, let $n$ be a natural number, $n>1$, which is square free and let $K=\mathbf{Q}(\sqrt{n})$. Then, if $\mathfrak{o}_{\mathrm{K}}$ is the ring of algebraic integers of $\mathrm{K}$, the group $\mathrm{SL}_{2}\left(\mathrm{o}_{\mathrm{K}}\right)$ operates in a natural way on $\mathscr{H} \times \mathscr{H}$ and on $\mathscr{H} \times \mathscr{H}^{-}$where $\mathscr{H}$ is the upper and $\mathscr{H}^{-}$the lower half plane of $\mathbf{C}$. The quotients of $\mathscr{H} \times \mathscr{H}$ and $\mathscr{H} \times \mathscr{H}^{-}$by the action of $\mathrm{SL}_{2}\left(0_{\mathrm{K}}\right)$ are the 2-dimensional complex spaces mentioned above. If the field $\mathrm{K}$ has a unit of negative norm, then the two actions on $\mathscr{H} \times \mathscr{H}$ and $\mathscr{H} \times \mathscr{H}^{-}$are isomorphic. This is true if $\mathrm{n}$ is a prime congruent $1 \bmod 4$, the only case we shall consider in this paper. Therefore, from now on we assume that $K=\mathbf{Q}(\sqrt{p})$, where $p$ is a prime congruent $1 \bmod 4$.

The complex space $\mathscr{H} \times \mathscr{H} / \mathrm{SL}_{2}\left(\mathrm{o}_{\mathrm{K}}\right)$ can be compactified by means of a finite number of points, called the cusps, to a compact 2-dimensional complex space. After resolving the cusps and also the quotient singularities on $\mathscr{H} \times \mathscr{H} / \mathrm{SL}_{2}\left(\mathrm{o}_{\mathrm{K}}\right)$, both in a canonical, explicit way, a nonsingular compact complex surface $Y(p)$ is obtained, which in fact is an algebraic surface. The field of meromorphic (i.e. rational) functions on $Y(p)$ is isomorphic to the field of meromorphic functions of $\mathscr{H} \times \mathscr{H} / \mathrm{SL}_{2}\left(\mathrm{o}_{\mathrm{K}}\right)$.

On the other hand, although no complete classification of algebraic surfaces is known, there exists a rough classification in several classes (for most of which the surfaces contained in that class can be classified completely, at least in principle). In big outline this classification was already known to the italian school, but its precise formulation (this time also covering the non-algebraic case) and many of the proofs involved are due to Kodaira. Now the question considered in this paper is the following: where are the surfaces $Y(p)$ to be placed in the rough classification of algebraic surfaces? 
Restricting ourselves to the case mentioned, where $n$ is a prime congruent $1 \bmod 4$, we are able to answer this question completely. This answer is embodied in Theorem IIl.1, which is our main result.

This paper is composed in the following way. Chapter I starts with generalities about surfaces, after which the rough classification of algebraic surfaces is explained (Theorem ROC). Chapter I contains furthermore a number of specific propositions, which are either used in Chapter III, or will be used in subsequent papers and are stated here for convenience. Some of the results proved here are already in the literature, or are even well known. They are included here for the reader's convenience, thus enabling him to understand many of the results of Chapter III without any further knowledge of the more refined parts of the theory of algebraic surfaces.

In Chapter II we collect some of the results of [12] which we need in Chapter III. Again for the readers convenience, we present them at some length and in a form suitable for our purposes, referring for details and general background always to [12], which paper also contains a more complete list of references.

As already said, the final Chapter III contains the statement and the proof of our results.

\section{Algebraic Surfaces}

Generalities. We shall consider divisors on a non-singular algebraic surface $X$, which is always supposed to be connected. Such a divisor is a finite sum $\sum n_{i} C_{i}, n_{i} \in \mathbf{Z}$, where $C_{i}$ is an irreducible algebraic curve on $X$. The divisor is called non-negative if all $n_{i}$ are non-negative, and it is called positive if it is non-negative and not zero. For any pair of divisors $D$ and $E$ on $X$, the symbol $D E$ will denote the intersection number of their homology classes, the surface being provided with its natural orientation. Sometimes we shall denote the homology class of a divisor $D$ also by $D$. The projective space of non-negative divisors linearly equivalent to $D$ will be denoted by $|D|$. Furthermore, $K_{X}$ or simply $K$ will stand for any canonical divisor, i.e. a divisor of a meromorphic 2

section of $\wedge \theta^{*}$, where $\theta^{*}$ is the covariant tangent bundle of $X$. As usual, we write $\mathcal{O}_{X}$ for the structure sheaf of $X$, and then set

$$
\operatorname{dim} H^{1}\left(X, \mathscr{O}_{X}\right)=q(X), \quad \text { the irregularity of } X
$$

(since $X$ is algebraic, $2 q(X)=b_{1}(X)$, the first betti number of $X$ );

$$
\begin{aligned}
& \operatorname{dim} H^{2}\left(X, \mathcal{O}_{X}\right)=p_{g}(X), \quad \text { the geometric genus of } X ; \\
& \operatorname{dim}\left|n K_{X}\right|+1=P_{n}(X), \quad \text { the } n \text {-th plurigenus of } X
\end{aligned}
$$

(by Serre duality, we have $p_{g}(X)=P_{1}(X)$ ). 
The arithmetic genus $\chi(X)$ of $X$ is the Euler-Poincaré characteristic of $X$ with coefficients in $\mathcal{O}_{X}([10]$, p. 151)

Thus by the Riemann-Roch theorem $([10], \S 20)$ we have

$$
\chi(X)=1-q(X)+p_{\mathrm{g}}(X) .
$$

The arithmetic genus is a birational invariant.

An exceptional curve on a non-singular complex surface is a nonsingular rational curve $E$ with $E^{2}=-1$. Blowing up a point means replacing that point by an exceptional curve, the result being another non-singular complex surface. Conversely, an exceptional curve can always be blown down, i. e. replaced by a point such that the result is again a non-singular complex surface $([16]$, p. 563). Blowing up or down (in) an algebraic surface gives again an algebraic surface ([15], p. 125).

Let $X$ be a non-singular compact complex surface, $Y$ the surface obtained by blowing up $p \in X, \rho: Y \rightarrow X$ the natural projection and $E=\rho^{-1}(p)$ the exceptional curve obtained.

Proposition I.1. With the notation just introduced, we have for every irreducible curve $C$ on $Y$ with $C \neq E$ :

$$
K_{Y} C=K_{X} \rho(C)+C E .
$$

Proof. If $\rho^{*}: H_{*}(X, \mathbf{Z}) \rightarrow H_{*}(Y, \mathbf{Z})$ denotes the Umkehr-homomorphism, then $([14]$, p. 31)

From this and

$$
K_{Y}=\rho^{*}\left(K_{X}\right)+E .
$$

the result follows.

$$
\rho^{*}\left(K_{X}\right) C=K_{X} \rho(C)
$$

For an irreducible curve $C$ on the non-singular compact complex surface $X$ one defines the virtual genus $\pi(C)$, in such a way that the adjunction formula

$$
K_{X} C=-C^{2}+2 \pi(C)-2
$$

holds ([15], p. 119). The virtual genus is always a non-negative integer. It is equal to the genus of $C$ if $C$ is non-singular, and strictly greater than the genus of the desingularisation of $C$ if $C$ is singular. Thus $\pi(C)$ vanishes if and only if $C$ is a non-singular rational curve. Also, $\pi(C)=1$ if and only if $C$ is a non-singular elliptic curve or a rational curve with exactly one cusp or ordinary double point.

Every non-singular algebraic surface $X$ can be obtained from a nonsingular algebraic surface $X_{0}$ without exceptional curves by a finite number of blowing-ups, i.e. there exists a finite number of non-singular algebraic surfaces $X_{0}, \ldots, X_{k}=X$ and points $p_{i} \in X_{i}$, such that for $i=1, \ldots, k$ the surface $X_{i}$ is obtained from $X_{i-1}$ by blowing up $p_{i-1}$. The surface $X_{0}$ is 
called a relatively minimal model of the surface $X$. In this paper we shall simply speak of a minimal model instead of a relatively minimal model, and surfaces without exceptional curves we shall call minimal surfaces. Minimal surfaces are classified by the following "rough classification" theorem ([19], Theorem 55).

Theorem ROC. Every algebraic surface without exceptional curves belongs to exactly one of the following classes:

1) the projective plane $\mathbf{P}_{2}\left(K^{2}=9\right)$,

2) the surfaces $\Sigma_{n}, n \geqq 0, n \neq 1\left(K^{2}=8\right)$,

3) the algebraic $\mathbf{P}_{1}$-bundles over a non-singular algebraic curve of genus $\geqq 1\left(K^{2} \leqq 0\right)$,

4) the algebraic $\mathrm{K}_{3}$-surfaces $\left(K^{2}=0\right)$,

5) the 2-dimensional algebraic tori $\left(K^{2}=0\right)$,

6) the honestly elliptic surfaces without exceptional curves $\left(K^{2}=0\right)$,

7) the surfaces of general type without exceptional curves $\left(K^{2} \geqq 1\right)$.

We now explain the terminology used in this theorem.

Let $\xi$ be the algebraic line bundle on $\mathbf{P}_{1}$ of degree 1 (i. e. the line bundle admitting holomorphic sections with exactly one simple zero). Then, for $\mathrm{n} \geqq 0$, the surface $\Sigma_{n}$ is defined as the total space of the projective bundle of $\xi \oplus \xi^{n+1}$. This bundle has a section with self-intersection $-\mathbf{n}$. (If it is blown down in $\Sigma_{1}$, the resulting surface is $\mathbf{P}_{2}$.) The surfaces of the classes 1) and 2) are exactly the rational surfaces without exceptional curves. (A surface is called rational if it is birationally equivalent to $\mathbf{P}_{2}$.) A $\mathrm{K}_{3}$-surface is by definition a surface $X$ with $q(X)=0$ and with trivial canonical bundle, i.e. $K_{X}=0$.

An elliptic surface is a surface which admits at least one elliptic fibring, i.e. a holomorphic map onto a non-singular curve such that all but a finite number of fibres are non-singular elliptic curves. Some of the surfaces in the classes 1)-5) and of their blow-ups admit elliptic fibrings. By an honestly elliptic surface we mean an elliptic surface not birationally equivalent to a surface from any of the classes 1)-5).

Finally, a surface of general type is a surface for which at least one $\mathrm{n}$-canonical system $|n K|, n \geqq 1$, provides a birational equivalence onto a (possibly singular) algebraic surface. If such a surface contains no exceptional curves, it is known ([3]) that, for $\mathrm{n}$ large enough, $|n K|$ gives an everywhere defined map onto the image. Furthermore, on $X$ there exists only a finite number of non-singular rational curves with selfintersection -2 . Let $A$ be the set of points through which passes at least one of these curves, and let $A_{1}, \ldots, A_{l}$ be the connected components of $A$. Then $|n K|, n \geqq 5$, provides a biregular map from $X-A$ onto its image, 
and each set $A_{i}$ is mapped into a normal singular point of the image, with different $A_{i}$ 's going to different points.

If a surface has a minimal model in class 1) or in class 2), it may have more minimal models, but these are all contained in classes 1) and 2). The surfaces of this sort are exactly the rational surfaces. If a surface has a minimal model in class 3), it may have more minimal models, but they are all contained in class 3 ). The surfaces of this sort are the ruled surfaces of genus $\geqq 1$.

If a surface has a minimal model in class 4 ), it has exactly one minimal model. So for such a surface we can speak of the minimal model. The same holds for surfaces which have a minimal model in either one of the classes 5), 6), and 7). The surfaces which have their minimal model in class 6) are exactly the honestly elliptic surfaces, and the surfaces with their minimal model in class 7) are exactly the surfaces of general type.

Remark. The finer classification of the surfaces within classes 1), 2),..., 6 ) is in principle known. For the "large" class 7) only some general facts are known.

The following results will be used in Chapter III to obtain the main result of this paper.

\section{Rational Surfaces}

Since the arithmetic genus is a birational invariant, all rational surfaces have arithmetic genus equal to 1 .

The following result is in essence due to $M$. Noether (see for example [19], p. 1053). We include an elementary proof of this well known fact, since only this result is needed for the proof of the rationality of $Y(5)$, $Y(13)$ and $Y(17)$ (see the proof of Theorem III.1).

Proposition I.2. Let $X$ be a non-singular algebraic surface with $q(X)=0$. If $X$ contains a non-singular rational curve $C$ with $C^{2} \geqq 0$, then $X$ is a rational surface.

Proof. From Proposition I.1 and the adjunction formula it follows that the self-intersection of a non-singular curve "goes down by one" if we blow up a point on the curve. Consequently, we can restrict ourselves to the case $C^{2}=0$. Let $\gamma$ be the line bundle on $X$ corresponding to $C$. Then there is an exact sequence of sheaves on $X([10], \mathrm{p} .130)$

$$
0 \rightarrow \mathcal{O}_{X} \rightarrow \Omega(\gamma) \rightarrow \Omega^{\prime}(\gamma \mid C) \rightarrow 0
$$

where $\Omega(\gamma)$ is the sheaf of local holomorphic sections of $\gamma$ and $\Omega^{\prime}(\gamma \mid C)$ the sheaf of local holomorphic sections of the restriction $\gamma \mid C$, extended by 0 outside $C$. Since the degree (first Chern number) of $\gamma \mid C$ is the self-intersection of $C([15]$, p. 117) the line bundle $\gamma \mid C$ is trivial, so

$$
\operatorname{dim} H^{0}\left(X, \Omega^{\prime}(\gamma \mid C)\right)=1 \text {. }
$$


Because $\operatorname{dim} H^{1}\left(X, \mathscr{O}_{X}\right)=q(X)=0$, we find $\operatorname{dim} H^{0}(X, \Omega(\gamma))=2$. The projective space of $H^{0}(X, \Omega(\gamma))$ can be identified with the set of zero divisors of holomorphic sections of $\gamma$. If we take two of these divisors, $C_{1}$ and $C_{2}$, with $C_{1} \neq C_{2}$, then two sections of $\gamma$ vanishing (with proper multiplicities) on $C_{1}$ and $C_{2}$ respectively, form a base of $H^{0}(X, \Omega(\gamma))$. Therefore, if $C_{1}$ and $C_{2}$ would have a strictly positive divisor $D$ in common, all sections of $\gamma$ would vanish on $D$, i.e. $D$ would be equal to $C$, which is impossible, since on $C$ there vanishes only a 1-dimensional space of sections of $\gamma$. Furthermore, from $C^{2}=0$ we deduce that two zero divisors never have any points in common. Hence the zero divisors of holomorphic sections of $\gamma$ are the fibres of a surjective holomorphic map $\rho: X \rightarrow \mathbf{P}_{1}$. From Bertini's theorem ([23],p.24) it follows that - except for a finite number of them - the fibres of $\rho$ are non-singular curves. The adjunction formula then implies that-except for a finite number of exceptions - the fibres are non-singular rational curves, the exceptions being exactly the reducible fibres. Again by the adjunction formula, these must have at least two different components. Let $\sum a_{i} C_{i}$ be such a fibre, where $a_{i}$ is the multiplicity of the component $C_{i}$. By an elementary case of Stein factorisation $\left([23]\right.$, p. 23) the fibre is connected. Therefore, from $\left(\sum a_{i} C_{i}\right) C_{i}=0$ we find that for all components $C_{i}$ the self-intersection is strictly negative. Also, from $\sum a_{i} K C_{i}=-2$ we conclude that there is at least one $C_{i}$, say $C_{i_{0}}$, with $K C_{i_{0}}<0$. The adjunction formula now gives $C_{i_{0}}^{2}=-1$, $\pi\left(C_{i_{0}}\right)=0$, i.e. $C_{i_{0}}$ is an exceptional curve. Repeating the argument, if necessary, for the surface which results from blowing down $C_{i_{0}}$, we find that $X$ is obtained by blowing up a surface $Y$ which admits a holomorphic map onto $\mathbf{P}_{1}$, everywhere of maximal rank, such that all the fibres on $Y$ are non-singular and rational. By an elementary argument ([1], p. 68) the surface $Y$ is an analytic $\mathbf{P}_{1}$-bundle over $\mathbf{P}_{1}$, and by another elementary argument the surface $Y$ is an algebraic $\mathbf{P}_{1}$-bundle over $\mathbf{P}_{1}$. Since every algebraic $\boldsymbol{P}_{1}$-bundle over $\mathbf{C}$ is algebraically trivial, $Y$ is birationally equivalent to $\mathbf{P}_{2}$. Hence also $X$ is a rational surface.

Theorem I.3 (Castelnuovo's Criterion). An algebraic surface $X$ is rational if and only if $q(X)=P_{2}(X)=0$.

For a proof see [19], p. 1052.

Proposition I.4. If on the non-singular algebraic surface $X$ with $q(X)=0$ there exists an irreducible curve $C$ with $K C<0$ and $C^{2} \geqq 0$, then $X$ is a rational surface.

Proof. Let $D \in|2 K|$. We set $D=a C+R$, with $a \geqq 0$ and $R C \geqq 0$. Then $2 K C=a C^{2}+R C \geqq 0$, which is a contradiction. Hence $|2 K|$ is empty, i.e. $P_{2}(X)=0$, and $X$ is rational by Castelnuovo's criterion. 
Proposition 1.5. Let $X$ be a non-singular algebraic surface with $q(X)=0$. If there exists on $X$ an irreducible curve $C$ with $K C<0$ and which has at least one singular point or which is not rational, then $X$ is rational. If there exists on $X$ an irreducible curve $C$ with $K C \leqq-2$, then again $X$ is a rational surface.

Proof. In both cases, the adjunction formula implies $C^{2} \geqq 0$. Hence $X$ is rational by Proposition I.4.

Proposition I.6. If on a non-singular algebraic surface $X$ with $q(X)=0$ there exist two intersecting exceptional curves, then $X$ is rational.

Proof. Let $E_{1}$ and $E_{2}$ be the exceptional curves with $E_{1} E_{2}>0$. If we blow down $E_{1}$, then on the blow-down surface $\tilde{X}$ the image $\tilde{E}_{2}$ of $E_{2}$ satisfies by Proposition I.1 the relation:

$$
\begin{aligned}
K_{\bar{X}} \tilde{E}_{2} & =K_{X} E_{2}-E_{1} E_{2} \\
& =-1-E_{1} E_{2} .
\end{aligned}
$$

Therefore, $\tilde{X}$ and hence $X$ is rational by Proposition I.5.

Elliptic Surfaces. Let $\rho: X \rightarrow R$ be an elliptic fibring of the non-singular algebraic surface $X$, and let $m_{1} F_{1}, \ldots, m_{l} F_{l}$ be the multiple fibres of $\rho$ $([16,17])$. Then, if $X$ is free of exceptional curves, the canonical divisor class on $X$ is given by

$$
K_{X}=\rho^{*}(A)+\sum_{i=1}^{l}\left(m_{i}-1\right) F_{i}
$$

where $A$ is a divisor class on $R$ of degree $\chi(X)-e(R)$, the number $e(R)$ being the Euler number of the curve $R$ (see [18], Theorem 12).

Proposition I.7. Let $X$ be a non-singular algebraic surface with $q(X)=0$ and $\chi(X)=2$. Suppose that on $X$ there exist irreducible curves $C$ and $D$ with $K C=C^{2}=0, K D \leqq 0$ and $C D=1$. Then $X$ is a blown up elliptic $\mathrm{K}_{3^{-}}$ surface.

Proof. The curve $C$ cannot intersect any exceptional curve $E$ on $X$, for after blowing down $E$ the image of $C$ would satisfy the conditions of Proposition I.4 and hence $X$ would be rational, contrary to the assumption that $\chi(X)=2$. In particular, $D$ is not an exceptional curve. Also $C$ itself is not an exceptional curve because $C^{2}=0$. Using Proposition $I .1$ we find therefore that after blowing down an exceptional curve on $X$, the resulting surface together with the images of $C$ and $D$ satisfies again our assumptions. So it is sufficient to prove the theorem for a surface $X^{\prime}$ containing no exceptional curves.

From the Riemann-Roch formula we find

$$
\operatorname{dim}|C|+\operatorname{dim}|K-C| \geqq 0 .
$$


Let $A \in|K-C|$. Then $A D<0$, and in particular $A \neq 0$. If we set $A=d D+R$, with $d \geqq 1$ and $R D \geqq 0$ we have $R C<0$. Consequently, if we set $R=e C+S$, with $e \geqq 1$ and $S C \geqq 0$, we find $(K-C) C=(d D+e C+S) C>0$, contrary to our assumptions. It follows that $\operatorname{dim}|K-C|=-1$ and $\operatorname{dim}|C| \geqq 1$. In the same way as in the proof of Proposition 1.2 we find that a 1-dimensional linear subspace of $|C|$, containing $C$, gives a holomorphic map $\rho$ : $X^{\prime} \rightarrow \mathbf{P}_{1}$. By construction $C$ is an irreducible non-multiple fibre of $\rho$. From Stein factorisation it follows that all but a finite number of fibres of $\rho$ are irreducible. By Bertini's theorem all but a finite number of fibres are non-singular, and by the adjunction formula therefore all but a finite number of fibres are non-singular elliptic. It follows that $\rho$ is an elliptic fibring of $X^{\prime}$. The curve $D$ intersects the irreducible fibre $C$ in one point transversally, hence $\rho$ has no multiple fibres. From the formula given above for the canonical class it follows that $K_{X^{\prime}}=0$, hence $X^{\prime}$ is an elliptic $\mathrm{K}_{3^{-}}$ surface, and $X$ is a blown up elliptic $\mathrm{K}_{3}$-surface.

(-2)-Configurations. Let $X$ be a complex surface. By a (-2)-configuration on $X$ we mean a configuration formed by a finite number of nonsingular rational curves $C_{1}, \ldots, C_{k}$, all of them with self-intersection -2 , such that $C_{1} \cup \cdots \cup C_{k}$ is a connected subset of $X$. The configuration formed by $C_{1}, \ldots, C_{k}$ will be denoted by $\left\{C_{1}, \ldots, C_{k}\right\}$ (any ordering). We shall not describe completely which $(-2)$-configurations can exist on the (blown up) surfaces of the classes 1 ), $\ldots, 7$ ) of Theorem ROC, but we shall prove a few facts about $(-2)$-configurations which will be used in Chapter III, and in forthcoming papers.

Let $X$ be a surface of general type without exceptional curves. As was mentioned before (after Theorem ROC) the holomorphic map, provided by $|n K|, \mathrm{n}$ sufficiently large, maps each (-2)-configuration on $X$ into a single point, with different configurations going to different points. The singularities thus obtainable are known: these are exactly the rational double points $A_{k}, D_{k}, E_{6}, E_{7}, E_{8}$ (see for example [4]). From this fact it follows which types of $(-2)$-configurations can exist on surfaces of general type without exceptional curves, and thus also which (-2)-configurations can exist on any surface of general type.

Using only the facts about surfaces of general type mentioned after Theorem ROC, we shall prove the following special result.

Proposition I.8. Let $X$ be a non-singular algebraic surface with $q(X)=0$. If on $X$ there exists a $(-2)$-configuration $\left\{D_{1}, \ldots, D_{k}\right\}$ such that the matrix $\left(D_{i} D_{j}\right)_{1 \leqq i, j \leqq k}$ is not negative definite, for example if

$$
\sum_{1 \leqq i<j \leqq k} D_{i} D_{j} \geqq k
$$

then $X$ is not a surface of general type, and hence, by Theorem $R O C, X$ is 
either rational, or a (blown up) $\mathrm{K}_{3}$-surface, or a (blown up) honestly elliptic surface.

Proof. To begin with, we shall prove: if some exceptional curve on $X$ intersects any of the curves $D_{1}, \ldots, D_{k}$, then $X$ is a rational surface. Indeed, if for any $i$ the intersection number $E D_{i} \geqq 2$, then after blowing down $E$, we find that $X$ is rational by Propositions I.1 and I.5. If $E$ intersects two of the curves $D_{1}, \ldots, D_{k}$, say $D_{1}$ and $D_{2}$, with $E D_{1}=E D_{2}=1$, then after blowing down $E$ we see that $X$ is rational by Proposition I.6. If for exactly one curve $D_{i}$, say $D_{1}$, we have $E D_{1}=1$, then after blowing down $E$ the image of $D_{1}$ is an exceptional curve. If $X$ is not rational, this curve intersects exactly one of the remaining curves $D_{2}, \ldots, D_{k}$. If $X$ is not rational, we can go on with the image of $D_{1}$ as we did before with $E$, and so on. This however implies that for a suitable ordering of the curves $D_{1}, \ldots, D_{k}$ we have $D_{1} D_{2}=\cdots=D_{k-1} D_{k}=1$, with all other $D_{i} D_{j}=0$. But this is contrary to the assumption that the matrix $\left(D_{i} D_{j}\right)_{1 \leqq i, j \leqq k}$ is not negative definite. Hence, if $X$ is not rational, then on a surface $Y$, without exceptional curves, from which $X$ is obtained by blowing up, there exists a (-2)configuration with an intersection matrix which is not negative definite. Then, if $X$ and thus $Y$ were of general type, by what is said about surfaces of general type after Theorem ROC, there would be a (-2)-configuration $\left\{D_{1}, \ldots, D_{k}, D_{k+1}, \ldots, D_{l}\right\}$ on $Y$, a neighbourhood $U$ of $\bigcup_{i=1}^{l} D_{i}$, a complex surface $V$ with exactly one normal singularity $p$, a holomorphic map $\rho: U \rightarrow V$ with $\rho\left(\bigcup_{i=1}^{l} D_{i}\right)=p$, such that $\rho$ would map $U-\bigcup_{i=1}^{l} D_{i}$ biregularly onto $V-p$. But then, according to a classical theorem of DuVal (see for example [20]) the matrix $\left(D_{i} D_{j}\right)_{1 \leqq i, j \leqq k}$ would be negative definite, contrary to the assumption.

Let us consider elliptic fibrings $\rho: X \rightarrow R$ of compact complex surfaces $X$ without exceptional curves. In [16] (p. 565) Kodaira has described all possible types of fibres of such fibrings. From Kodaira's results it follows in particular which (-2)-configurations occur as (set theoretic) fibres in elliptic fibrings of such surfaces $X$. Below we give a list of these configurations, using Kodaira's notation. We point out that in this list we consider only the underlying $(-2)$-configurations, thus forgetting about the multiplicities of the components.

In the following enumeration $C_{i}$ always denotes a non-singular rational curve with $C_{i}^{2}=-2$. By $C_{i} \circ C_{j}=\sum_{i=1}^{m} \alpha_{i} p_{i}$ we indicate that the curves $C_{i}$ and $C_{j}$ intersect precisely in points $p_{1}, \ldots, p_{m}\left(p_{i} \neq p_{j}\right.$ for $\left.i \neq j\right)$ with multiplicity $x_{1}, \ldots, x_{m}$ respectively. If an intersection $C_{i}{ }^{\circ} C_{j}$ does not occur it is understood to be empty. 


\begin{tabular}{lll}
\hline Fibre type & Curves & Intersection behaviour \\
\hline $\mathrm{m} \mathrm{I}_{2}$ & $C_{1}, C_{2}$ & $C_{1} \circ C_{2}=p_{1}+p_{2}$ \\
$\mathrm{III}$ & $C_{1}, C_{2}$ & $C_{1} \circ C_{2}=2 p_{1}$ \\
$\mathrm{IV}$ & $C_{1}, C_{2}, C_{3}$ & $C_{1} \circ C_{2}=C_{1} \circ C_{3}=C_{2} \circ C_{3}=p_{1}$ \\
$\mathrm{mI}_{k}$ & $C_{1}, \ldots, C_{k}(k \geqq 3)$ & $C_{1} \circ C_{i+1}=p_{i}(i=1, \ldots, k-1), C_{k} \circ C_{1}=p_{0}$ \\
$\mathrm{I}_{k}^{*}$ & $C_{1}, C_{2}, C_{3}, C_{4}, C_{5}$, & $C_{1} \circ C_{5}=p_{1}, C_{2} \circ C_{5}=p_{2}, C_{3} \circ C_{k+5}=p_{3}$, \\
& $\ldots, C_{k+5}$ & $C_{4} \circ C_{k+5}=p_{4}, C_{5} \circ C_{6}=p_{5}, \ldots, C_{k+4} \circ C_{k+5}=p_{k+4}$ \\
II* & $C_{1}, \ldots, C_{9}$ & $C_{1} \circ C_{2}=p_{1}, \ldots, C_{5} \circ C_{6}=p_{5}$, \\
& & $C_{6} \circ C_{7}=p_{6}, C_{6} \circ C_{8}=q_{6}, C_{7} \circ C_{9}=p_{7}$ \\
III* & $C_{1}, \ldots, C_{8}$ & $C_{1} \circ C_{2}=p_{1}, C_{2} \circ C_{3}=p_{2}, C_{3} \circ C_{4}=p_{3}$, \\
& & $C_{4} \circ C_{5}=p_{4}, C_{4} \circ C_{6}=q_{4}, C_{5} \circ C_{7}=p_{5}$, \\
& & $C_{7} \circ C_{8}=p_{7}$ \\
IV* & $C_{1}, \ldots, C_{7}$ & $C_{1} \circ C_{2}=p_{1}, C_{2} \circ C_{3}=p_{2}, C_{3} \circ C_{4}=p_{3}$, \\
& & $C_{3} \circ C_{5}=q_{3}, C_{4} \circ C_{6}=p_{4}, C_{5} \circ C_{7}=p_{5}$ \\
\hline
\end{tabular}

The (-2)-configurations occurring in this list will be called the elliptic (-2)-configurations.

Proposition 1.9. Let $X$ be a non-singular algebraic surface with $q(X)=0$ and $\chi(X) \geqq 2$. Suppose that on $X$ there exists a $(-2)$-configuration $\left\{C_{1}, \ldots\right.$, $\left.C_{k}, C\right\}$, such that $\left\{C_{1}, \ldots, C_{k}\right\}$ is an elliptic (-2)-configuration, but the whole configuration $\left\{C_{1}, \ldots, C_{k}, C\right\}$ is not. Then $X$ is a (blown up) elliptic $\mathrm{K}_{3}$-surface.

Proof. From $\chi(X) \geqq 2$ it follows that $X$ is not rational. Using this fact we find in a way quite similar to the first part of the proof of Proposition I.8 that no exceptional curve on $X$ intersects any of the curves $C_{1}, \ldots, C_{k}, C$. Consequently, it is sufficient to consider the case that $X$ contains no exceptional curves. By the definition of an elliptic ( -2$)$-configuration there exist strictly positive integers $a_{1}, \ldots, a_{k}$, such that $\left(\sum a_{i} C_{i}\right)^{2}=0$. (Since we shall use it later on, we shall assume-as of course we may-that $\operatorname{GCD}\left(a_{1}, \ldots, a_{k}\right)=1$.) Therefore, by the theorem of DuVal (loc. cit.), $X$ is not a surface of general type, and hence $X$ is either honestly elliptic or a $\mathrm{K}_{3}$-surface.

Now let $\rho: X \rightarrow \mathbf{P}_{1}$ be an elliptic fibring of $X$, and let $m \sum_{j=1}^{l} m_{j} F_{j}$, with $\operatorname{GCD}\left(m_{1}, \ldots, m_{l}\right)=1$, be a fibre of $\rho$. According to a theorem of Zariski ([23], p. 165) we have for all integers $c_{1}, \ldots, c_{l}$ the inequality $\left(\sum_{j=1}^{l} c_{j} F_{j}\right)^{2} \leqq 0$, and the equality $\left(\sum_{j=1}^{l} c_{j} F_{j}\right)^{2}=0$ holds if and only if there exists an integer $p$ such that $c_{j}=p m_{j}, j=1, \ldots, l$. Since there exist strictly positive integers $a_{1}, \ldots, a_{l}$ such that $\left(\sum a_{i} C_{i}\right)^{2}=0$ it follows that at least one of the curves $C_{1}, \ldots, C_{k}, C$ has a strictly positive intersection with the 
fibres of $\rho$. From $\chi(X) \geqq 2$, combined with $K_{X} C_{1}=\cdots=K_{X} C_{k}=K_{X} C=0$ and with the formula for $K_{X}$ given earlier, it follows that $K_{X}=0$. Hence in any case $X$ is a $K_{3}$-surface. To finish the proof we still have to show that $X$ is elliptic. In fact, from the Riemann-Roch formula we derive that $\operatorname{dim}\left|\sum a_{i} C_{i}\right| \geqq 1$. From the theorem of Zariski just used it follows that no 1-dimensional linear subspace of $\left|\sum a_{i} C_{i}\right|$, containing $\sum a_{i} C_{i}$ has a fixed component. Finally, in the same way as in the proof of Proposition I.7 we conclude that such a linear subspace gives an elliptic fibring of $X$.

\section{Curves on the Hilbert Modular Surfaces}

The Quotient Singularities. As has been announced already in the introduction, we recall in this Chapter only the facts we shall need later on. For more details we refer to [12].

Let $\mathrm{K}$ be the real quadratic field $\mathbf{Q}(\sqrt{\mathrm{p}})$, where $\mathrm{p}$ is a prime congruent $1 \bmod 4$, and let $v$ be its ring of integers. If $\mathscr{H}$ is the upper half plane in $\mathrm{C}$, then $\mathrm{SL}_{2}(\mathfrak{o})$ acts on $\mathscr{H}^{2}=\mathscr{H} \times \mathscr{H}$ by

$$
\left(\begin{array}{ll}
a & b \\
c & d
\end{array}\right)\left(z_{1}, z_{2}\right)=\left(\frac{a z_{1}+b}{c z_{1}+d}, \frac{a^{\prime} z_{2}+b^{\prime}}{c^{\prime} z_{2}+d^{\prime}}\right)
$$

where $\mathrm{a}^{\prime}, \mathrm{b}^{\prime}, \ldots$ are the conjugates of $\mathrm{a}, \mathrm{b}, \ldots$ over $\mathbf{Q}$. The Hilbert modular group $\mathrm{G}=\mathrm{SL}_{2}(\mathbf{0}) /\{+1,-1\}$ acts effectively on $\mathscr{H}^{2}$. The orbit space $\mathscr{H}^{2} / \mathrm{G}$ is a complex space of dimension 2 with finitely many quotient singularities. For a suitable $r$, such a singularity is locally the quotient of $\mathbf{C}^{2}$ by a linear action of the group of $r$-th roots of unity, acting freely outside the origin. If for $\left(z_{1}, z_{2}\right) \in \mathbf{C}^{2}$ and $\xi^{r}=1$ this action is of the form

$$
\xi\left(z_{1}, z_{2}\right)=\left(\xi z_{1}, \xi^{\mathrm{q}} z_{2}\right)
$$

where $q$ and $r$ are relatively prime, we shallsay that the quotient singularity is of order $r$ and of type $(r ; 1, q)$.

Prestel ([21]) has proved the following proposition.

Proposition II.1. Let $\mathrm{a}_{\mathrm{r}}$ be the number of quotient singularities of order $\mathrm{r}$ in $\mathscr{H}^{2} / \mathrm{G}$, where $\mathrm{G}$ is the Hilhert modular group for the field $\mathrm{K}=\mathbf{Q}(\sqrt{\mathrm{p}})$ and where $\mathrm{p}$ is a prime congruent $1 \bmod 4$. Then we have

$$
\begin{aligned}
& \text { for } \mathrm{p}=5: \mathrm{a}_{2}=\mathrm{a}_{3}=\mathrm{a}_{5}=2, \quad \mathrm{a}_{\mathrm{r}}=0 \quad \text { for } \mathrm{r}>5, \\
& \text { for } \mathrm{p}>5: \mathrm{a}_{2}=\mathrm{h}(-\mathrm{p}), \quad \mathrm{a}_{3}=\mathrm{h}(-3 \mathrm{p}), \quad \mathrm{a}_{\mathrm{r}}=0 \quad \text { for } \mathrm{r} \geqq 5
\end{aligned}
$$

Here $h$ (b) denotes the class number of the field $Q(\sqrt{b})$. The numbers $a_{t}$ are even. Half of the singularities of order 3 are of type $(3 ; 1,1)$ (the first kind) and half of them are of type $(3 ; 1,2)$ (the second kind). For $p=5$, the quotient singularities of order 5 are of type $(5 ; 1,3)$. 
The minimal resolutions of the quotient singularities of $\mathscr{H}^{2} / \mathrm{G}$ can be described by the following diagrams. In these diagrams the lines indicate non-singular rational curves, and the numbers are their self-intersection numbers. The intersection of different curves is transversal.

Quotient singularity of order 2:

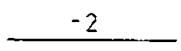

Quotient singularity of order 3 and of the first kind:

Quotient singularity of order 3 and of the second kind:

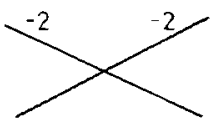

Quotient singularity of type $(5 ; 1,3)$ :

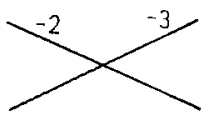

The Euler number of $\mathscr{H}^{2} / \mathrm{G}$ is given by

$$
e\left(\mathscr{H}^{2} / \mathrm{G}\right)=2 \zeta_{\mathrm{K}}(-1)+\sum_{\mathrm{r} \geqq 2} \mathrm{a}_{\mathrm{r}} \frac{\mathrm{r}-1}{\mathrm{r}}
$$

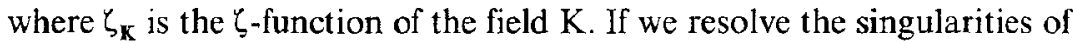
$\mathscr{H}^{2} / \mathrm{G}$ by way of a minimal resolution we obtain from $\mathscr{H}^{2} / \mathrm{G}$ a (noncompact) non-singular complex surface $X(p)$. Blowing up a quotient singularity increases the Euler number by the number of curves occuring in the resolution. Therefore, by Proposition II.1 and formula (1) we abtain

Proposition II.2. If $\mathrm{G}$ is the Hilbert modular group for the field $\mathrm{K}=\mathbf{Q}(\sqrt{\mathrm{p}})$, then the minimal desingularisation $X(p)$ of $\mathscr{H}^{2} / \mathrm{G}$ has the following Euler number

$$
\begin{aligned}
& e(X(5))=13 \\
& e(X(p))=2 \zeta_{\mathrm{K}}(-1)+\frac{3}{2} \mathrm{~h}(-\mathrm{p})+\frac{13}{6} \mathrm{~h}(-3 \mathrm{p}), \quad \text { for } \mathrm{p}>5 .
\end{aligned}
$$

The Cusps. The manifold $X(p)$ can be compactified by finitely many "cusps", which are in one-to-one correspondence with the ideal classes 
of the ring of algebraic integers in the field $K=\mathbf{Q}(\sqrt{p})$. The compactified space $\overline{X(p)}$ has $h(p)$ singular points, corresponding to the cusps. If we replace each of these singular points by a minimal resolution, we obtain a non-singular algebraic surface $Y(p)$. These surfaces are the Hilbert modular surfaces as they appear in the Introduction.

It has been shown in [12] that the singular points of $\overline{X(p)}$ admit a cyclic resolution. Since in our case (p prime congruent $1 \bmod 4)$ the ring 0 has a unit of negative norm, in $\mathfrak{o}$ the groups $\mathfrak{C}$ of wide ideal classes and $\mathfrak{C}^{+}$ of narrow ideal classes coincide. The squaring operation Sq: $\mathfrak{n} \rightarrow \mathfrak{n}^{-2}$ from $\mathfrak{C}$ to $\mathfrak{C}^{+}$is bijective, because $h(p)=|\mathfrak{C}|=\left|\mathfrak{C}^{+}\right|$is odd $([12], 3.7)$. Therefore the cusps are of type $\left(\mathfrak{M}, \mathfrak{U}^{2}\right)$, where $\mathfrak{U}$ is the group of units of $\mathfrak{p}$ and $\mathfrak{M}$ runs through a complete system of representatives of the ideal classes of $\mathfrak{v}$. The group $\mathfrak{l}^{2}$ equals the infinite cyclic group $\mathfrak{U}^{+}$of totally positive units. Thus the result on the desingularisation of the $h(\mathrm{p})$ singular points of $\overline{X(p)}$ can be formulated in the following way.

A real quadratic irrationality $w$ is called reduced if

$$
0<\mathrm{w}^{\prime}<1<\mathrm{w}
$$

where $w^{\prime}$ is again the conjugate of w over $\mathbf{Q}$. A real quadratic irrationality $w$ is reduced if and only if its continued fraction of the form

$$
w=a_{0}-\frac{1}{a_{1}-\frac{1}{\cdots}} \quad\left(a_{i} \in Z, a_{i} \geqq 2 \text { for } i \geqq 1\right)
$$

is purely periodic, i.e. there exists a natural number $r>1$, such that that $a_{i}=a_{i+r}$ for $i \geqq 0$. A quadratic irrationaliy $w$ has discriminant $p$ if

$$
w=\frac{M+\sqrt{p}}{2 N}
$$

where $M, N$ are integers, $N>0$ and $M^{2}-p$ congruent $0 \bmod 4 N$. Let $\mathrm{L}(\mathrm{p})$ be the set of all reduced quadratic irrationalities of discriminant $\mathrm{p}$. The set $\mathrm{L}(\mathrm{p})$ is finite, and its cardinality will be denoted by $\ell(\mathrm{p})$. If $w \in \mathrm{L}(\mathrm{p})$ and

$$
\mathrm{w}=[\mathrm{w}]+1-\frac{1}{\mathrm{w}_{1}}=\mathrm{b}-\frac{1}{\mathrm{w}_{1}}
$$

then $w_{1} \in L(p)$. Thus for each $w \in L(p)$ we have a successor $w_{1} \in L(p)$. In this way, the finite set $L(p)$ is arranged in finitely many cycles, which are disjoint. Alternatively, we can say that $\mathbf{Z}$ acts on L(p) with finitely many orbits. Formula (5) is the beginning of the continued fraction for $w$. The $a_{0}$ of $(3)$ equals $b$, and we have $b \geqq 2$. The following proposition is a reformulation of results in [12], $\S 2$ and $\S 3$. 
Proposition II.3. The number of cycles in $\mathrm{L}(\mathrm{p})$ equals the class number $\mathrm{h}(\mathrm{p})$. There is a natural correspondence between the singular points of $\overline{X(p)}$ and the cycles in $\mathrm{L}(\mathrm{p})$. To each $\mathrm{w} \in \mathrm{L}(\mathrm{p})$ one can associate a rational curve $S_{x}$ occurring in the minimal resolution of that singular point of $\overline{X(p)}$ which corresponds to the cycle of $\mathrm{w}$. The relationship thus established between the curves of the minimal resolution of a singular point of $\overline{X(p)}$ and the elements of the corresponding cycle in $\mathrm{L}(\mathrm{p})$ is one to one.

For $\mathrm{p}=5$, we have $\ell(\mathrm{p})=1$, and the unique reduced quadratic irrationality is $\mathrm{w}=\frac{3+\sqrt{5}}{2}=3-\frac{1}{\mathrm{w}}$. In this case the curve $S_{w}$ is a rational curve with one singularity, an ordinary double point, and $S_{w}^{2}=-1$.

For $\mathrm{p}>5$, the curves $S_{w}$ are all non-singular and their self-intersection number is $S_{w}^{2}=-[\mathrm{w}]-1$. Furthermore, we have $\ell(\mathrm{p}) \geqq 3$, and in fact each cycle has a length $\geqq 3$. Each curve $S_{w}$ intersects only its successor and its predecessor, each of them transversally in exactly one point.

Let $w_{0}=\frac{\{\sqrt{p}\}+\sqrt{p}}{2}$, where $\{\sqrt{p}\}=2 a+1$ is the smallest odd integer greater than $\sqrt{\mathrm{p}}$. The cycle of $\mathrm{w}_{0}$ corresponds to the ideal class of the principal ideals of $\mathbf{o}$. The successor of $w_{0}$ will be called $w_{1}$, its predecessor $w_{-1}$, and so on, until for a certain $t$ the curves $S_{w_{t}}$ and $S_{w-t}$ intersect. The curves $S_{w_{t}}$ will simply be denoted by $S_{i}$. If we put

$$
\mathrm{b}_{\mathrm{i}}=\left[\mathrm{w}_{\mathrm{i}}\right]+1 \text {, }
$$

then we have

$$
S_{i}^{2}=S_{-i}^{2}=-\mathrm{b}_{\mathrm{i}}=-\mathrm{b}_{-\mathrm{i}}
$$

In particular,

$$
S_{0}^{2}=-\mathrm{b}_{0}=-\{\sqrt{\mathrm{p}}\}=-(2 \mathrm{a}+1) .
$$

As observed before, the resolution of the cusp, corresponding to the cycle of $\mathrm{w}_{0}$ looks as follows

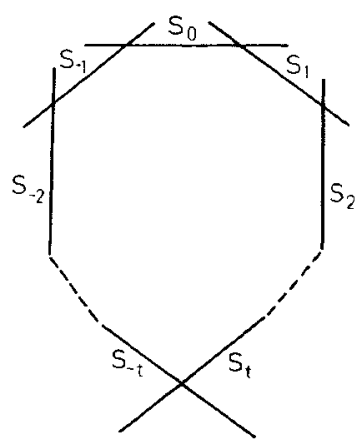


If we denote continued fractions of the form (3) by

we have

$$
\left[\left[\mathrm{a}_{0}, \mathrm{a}_{1}, \mathrm{a}_{2}, \ldots\right]\right]
$$

$$
\mathrm{w}_{0}=\left[\left[\mathrm{b}_{0}, \mathrm{~b}_{1}, \ldots, \mathrm{b}_{\mathrm{t}}, \mathrm{b}_{\mathrm{t}}, \ldots, \mathrm{b}_{1}\right]\right]
$$

where the bar indicates the period. Observe that $b_{t+1}=b_{-t}=b_{t}$, etc.

The first prime $p$ congruent $1 \bmod 4$ with $h(p)>1$ is $p=229$. If we want to tabulate all elements of $L(p)$ for $p<229$, we only have to consider the cycle of $w_{0}$. We put

$$
\mathrm{w}_{\mathrm{i}}=\frac{\mathrm{M}_{\mathrm{i}}+\sqrt{\mathrm{p}}}{2 \mathrm{~N}_{\mathrm{i}}} \text {. }
$$

It is easy to show that $\mathrm{N}_{-i}=N_{i}$. Therefore, we shall tabulate $b_{i}$ and $N_{i}$ only for $0 \leqq i \leqq t$. The $M_{i}$ we do not need. Table 1 (p. 22) gives the $b_{i}$ and $\mathrm{N}_{\mathrm{i}}$ for $\mathrm{p} \leqq 73$, whereas in Table 2 the numbers $\ell(\mathrm{p})$ can be found for $29 \leqq \mathrm{p} \leqq 317$.

The boundary $V$ of a suitable compact neighbourhood $U$ in $Y(p)$ of the union $S$ of all curves $S_{w}$ is a 3-dimensional manifold with $\mathrm{h}(\mathrm{p})$ connectedness components, each of which is a torusbundle over a circle. The neighbourhood $U$ has $S$ as a deformation retract. Therefore, the betti numbers of $U$ are given by: $b_{0}(U)=b_{1}(U)=\mathrm{h}(\mathrm{p}) ; b_{2}(U)=f(\mathrm{p})$, $b_{\mathrm{i}}(U)=0$ for $\mathrm{i} \geqq 3$. The Euler number $e(U)$ equals $\ell(\mathrm{p})$. The complement in $Y(p)$ of the interior of $U$ is a compact manifold, with boundary $V$, which is a deformation retract of $X(p)$. A simple additivity property of the Euler number $(e(A \cup B)=e(A)+e(B)-e(A \cap B))$ implies in virtue of $e(V)=0$ the formula

$$
e(Y(p))=e(X(p))+\ell(\mathrm{p}) .
$$

Since $b_{1}(Y(p))=0$, formula (7) enables us to calculate the second betti number of the surface $Y(p)$. For the sake of completeness we indicate a proof of the fact that $b_{1}(Y(p))$ vanishes; for more details we refer to [6] (see also [5], Teil I, Satz 8).

Proposition II.4. The Hilbert modular surfaces are regular, i.e. their first betti number $b_{1}(Y(p))$ vanishes.

Proof. We remove from $\mathscr{H}^{2}$ suitable open balls around the points with non trivial isotropy groups. The resulting space $\left(\mathscr{H}^{2}\right)^{\prime}$ is simply-connected, and $\left(\mathscr{H}^{2}\right)^{\prime} / \mathrm{G}$ is a (non compact) manifold with a boundary consisting of finitely many lens spaces. According to Serre ([24]) the abelianised group $\mathrm{G} /[\mathrm{G}, \mathrm{G}]$ is finite. Therefore, the first betti number of $\left(\mathscr{H}^{2}\right)^{\prime} / \mathrm{G}$ and also of $X(p)$ vanishes. The last fact follows because the tubular neighbourhoods of the configuration of curves into which a quotient singularity is blown up, are simply connected. Therefore, $b_{1}(\overline{Y(p)-U})$ vanishes. Each one-dimensional homology class of $U$ can be represented 
by a cycle in $U-S$, and thus by a cycle in $\partial U=V$. The van Kampen-

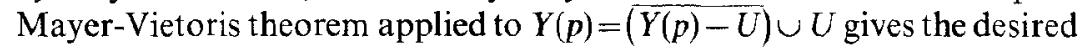
result,

The Curves $F_{N}$. Next we recall some facts about the curves $F_{N}$ on the surfaces $Y(p)([12], \S 4)$.

A natural number $\mathrm{N}$ is called admissible with respect to $\mathrm{p}$ if $\mathrm{N}$ is not congruent 0 mod $p^{2}$ and if $N$ does not contain any prime number $q \neq p$ with $\left(\frac{\mathrm{q}}{\mathrm{p}}\right)=-1$, i. e. all the primes contained in $\mathrm{N}$ are quadratic residues $\bmod \mathbf{p}$. Thus in $\mathbf{v} \subset \mathbf{Q}(\sqrt{\mathrm{p}})$ all these primes are equal to a product of two different prime ideals $q, q^{\prime}$, where $\mathrm{x} \rightarrow \mathrm{x}^{\prime}$ denotes as before the non-trivial automorphism of the field $K=\mathbf{Q}(\sqrt{\mathrm{p}})$.

For every admissible $\mathrm{N}$ there is defined an algebraic curve $F_{N}$ on the surface $Y(p)$. It is always irreducible, but may have singularities. For different admissible numbers $\mathrm{N}_{1}, \mathrm{~N}_{2}$ the corresponding curves $F_{N_{1}}$ and $F_{\mathrm{N}_{2}}$ are also different. We do not recall the definition of the curves $F_{N}$ in full generality, but restrict ourselves to the following special case.

If $\mathrm{N}=\mathrm{jj}$, where $\mathrm{j}$ is a totally positive integer in $\mathrm{K}$ which is not divisible by any natural number $\neq 1$, then $\mathrm{N}$ is admissible, and the curve

$$
\begin{aligned}
& z_{1}=j t \\
& z_{2}=j^{\prime} t, \quad t \in \mathscr{H}
\end{aligned}
$$

on $\mathscr{H}^{2}$ projects down to a curve in $\mathscr{H}^{2} / \mathrm{G}$. The closure in $\mathscr{H}^{2} / \mathrm{G}$ of this last curve is an algebraic curve on $Y(p)$, and this curve is precisely $F_{N}$.

If the class number $h(p)$ of the field $K$ equals 1 , then any admissible $N$ can be written in the form $N=j j^{\prime}$, where $\mathrm{j}$ has the properties mentioned above.

The curve $F_{N}$ has a non-singular model $\overline{\mathscr{H}} / \Gamma$, where $\Gamma$ is a discrete group acting on $\mathscr{H}$ and where the bar indicates the compactification of $\mathscr{H} / \Gamma$ by the finitely many cusps of $\Gamma$. If $\mathrm{N}$ is not congruent $0 \bmod \mathrm{p}$, then $\Gamma=\Gamma_{0}(\mathrm{~N})$, where

$$
\Gamma_{0}(N)=\left\{\left(\begin{array}{ll}
a & b \\
c & d
\end{array}\right) \in \operatorname{SL}_{2}(Z) ; c \text { congruent } 0 \bmod N\right\} .
$$

If $\mathrm{N}=1$, then $\Gamma=\mathrm{SL}_{2}(\mathrm{Z})$, and the curve $F_{1}$ comes from the ordinary diagonal $z_{1}=z_{2}$ in $\mathscr{H}^{2}$.

If $N=p$, then $\Gamma=\Gamma_{*}(p)$ which group is the extension of index 2 of $\Gamma_{0}(p)$ by the element

$$
\left(\begin{array}{cc}
0 & p^{\frac{1}{2}} \\
p^{-\frac{1}{2}} & 0
\end{array}\right)
$$


The curve $F_{p}$ can be given in the form (8) with $\mathrm{j}=\sqrt{\mathrm{p}} \mathrm{e}_{0}$, where $\mathrm{e}_{0}$ is a positive unit of negative norm $\left(\mathrm{e}_{0}^{\prime}<0\right)$. In our case such a unit always exists, as was already mentioned before. Also the equations $z_{1}=e_{0}^{2} z_{2}$ or $z_{1} z_{2}=-1$ give rise to the curve $F_{p}$.

There is a natural map

$$
\overline{\mathscr{H}} \bar{\Gamma}-\mathscr{H} / \Gamma \rightarrow S
$$

which indicates where the curve $F_{N}$ passes through the curves $S_{w}$. The (finite) number of points in $\overline{\mathscr{H}} / \bar{\Gamma}-\mathscr{H} / \Gamma$, i.e. the number of cusps of $\Gamma$ will be denoted by $\sigma(\Gamma)$. There are well known formulae for $\sigma(\Gamma)$. The number $\sigma(\Gamma)$ equals $2^{r}$ if $\mathrm{N}$ is a product of $\mathrm{r}$ different primes $(\neq \mathrm{p})$, it equals 1 if $\mathrm{N}=1$ or $\mathrm{N}=\mathrm{p}$. Some information about the map (9) is contained in the following proposition.

Proposition II.5. If the reduced quadratic irrationality $\mathrm{w} \in \mathrm{L}(\mathrm{p})$ is of the form

$$
\mathrm{w}=\frac{\mathrm{M}+\sqrt{\mathrm{p}}}{2 \mathrm{~N}}
$$

then the curve $F_{N}$ intersects $S_{w}$ transversally in a point which is not a double point of $S$.

The curve $F_{1}$ intersects $S_{0}$ transversally and this is the only intersection of $F_{1}$ with $S$.

The curve $F_{p}$ passes through the intersection point of $S_{t}$ and $S_{-t}$, intersecting $S_{t}$ and $S_{-t}$ transversally. This is the only intersection of $F_{p}$ and $S$.

To describe the basic configuration of curves on $Y(p)$ as we need it for our results on classification we have to recall the properties of the involution $l$ on $\mathscr{H}^{2} / \mathrm{G}$, induced by the involution $\left(\mathrm{z}_{1}, \mathrm{z}_{2}\right) \rightarrow\left(\mathrm{z}_{2}, \mathrm{z}_{1}\right)$ of $\mathscr{H}^{2}$ (compare [12], 5.4, where this involution is called T). The involution $t$ acts on the quotient sigularities of $\mathscr{H}^{2} / \mathrm{G}$. The description of this action ([22]) depends on the residue class of $\mathrm{p}$ mod 24. Therefore we define:

$$
\begin{array}{lll}
\varepsilon=1 & \text { for } p \text { congruent } 1 \bmod 3, & \varepsilon=0 \text { for } p \text { congruent } 2 \bmod 3 \\
\delta=1 \text { for } p \text { congruent } 1 \bmod 8, & \delta=0 \text { for p congruent } 5 \bmod 8 .
\end{array}
$$

Now consider $F_{1}$ and $F_{p}$ as curves on $\mathscr{H}^{2} / \mathrm{G}$. Then the following holds on $\mathscr{H}^{2} / \mathrm{G}$ :

$F_{1}$ and $F_{p}$ are both pointwise fixed under $t$.

Of the $\mathrm{h}(-\mathrm{p})$ quotient singularities of order 2 , half of them lie on $F_{p}$, and not on $F_{1}$, and one of them (represented by $(\mathrm{i}, \mathrm{i}) \in \mathscr{H}^{2}$ ) lies on $F_{1}$ and $F_{p}$, and is the only intersection point of $F_{1}$ and $F_{p}$ in $\mathscr{H}^{2} / \mathrm{G}$. There are in addition $\delta$ quotient singularities of order 2 which are fixed under $l$. "They" lie neither on $F_{1}$ nor on $F_{p}$. The remaining order 2 singularities are interchanged pairwise under $l$. Of the $h(-3 p)$ quotient singularities of 
order 3 , exactly half of them are of type $(3 ; 1,2)$. They lie on $F_{p}$. There is one singularity of type $(3 ; 1,1)$, represented by $(\mathrm{r}, \mathrm{r})$, where $\mathrm{r}=-\frac{1}{2}+\frac{1}{2} \mathrm{i} \sqrt{3}$, which lies on $F_{1}$, whereas $\varepsilon$ such singularities lie on $F_{p}$. The remaining singularities of type $(3 ; 1,1)$ are interchanged pairwise. The involution acts freely outside $F_{1}$ and $F_{p}$ and the quotient singularities of order 2 and 3 .

The involution $l$ can be carried over (lifted and extended) to $Y(p)$. Since $F_{1}$ and $F_{p}$ are pointwise fixed under $l$ it follows from general theorems about involutions on complex surfaces that $F_{1}$ and $F_{p}$ are nonsingular curves on $Y(p)$, which do not intersect on $Y(p)$.

On $Y(p)$ we have the following basic configuration. (In this diagram we omit the curves coming from the cusps belonging to non principal ideal classes, and also the curves coming from quotient singularities which are pairwise interchanged.)

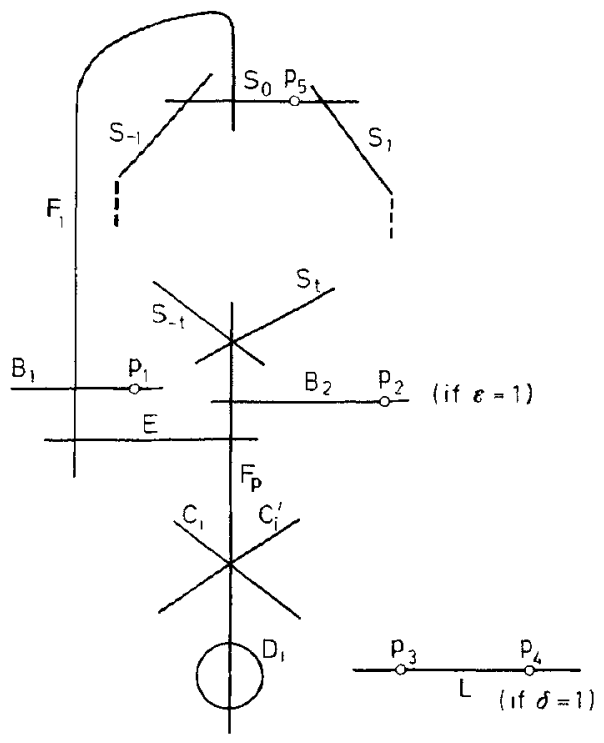

The basic configuration

The involution $t$ interchanges the curves $S_{j}$ and $S_{-j}$. The curves $B_{1}$ and $B_{2}$ come from the resolution of the quotient singularities of type $(3 ; 1,1)$ which lie on $F_{1}$ and $F_{p}$ respectively (the curve $B_{2}$ exists only if $\varepsilon=1$ ), and have both self-intersection number -3 . The curve $E$ comes from the quotient singularity of order 2 which lies on both $F_{1}$ and $F_{p}$. The curve $L$ (which exists only if $\delta=1$ ) comes from the quotient singularity of order 2 which is fixed under $t$. The curves $C_{i}, C_{i}^{\prime}$ represent the resolutions of the $\frac{\mathrm{h}(-3 \mathrm{p})}{2}$ quotient singularities of type $(3 ; 1,2)$; they are interchanged 
by 1 . The curves $D_{i}$ are the resolutions of the $\frac{\mathrm{h}(-\mathrm{p})}{2}$ quotient singularities of order 2 which lie on $F_{p}$. The curves $E, L, C_{i}, C_{i}^{\prime}$ and $D_{i}$ all have selfintersection number -2 . The curves $B_{1}, B_{2}, E, D_{i}$ and $L$ are mapped to themselves by $t$. The points $p_{1}, p_{2}, p_{3}, p_{4}$ and $p_{5}$ are isolated fixed points of $t$. On $Y(p)$ the involution $t$ has exactly $2+\varepsilon+2 \delta$ isolated fixed points.

To every normal isolated singular point of a 2-dimensional complex space one can attach a Chern divisor. In extenso ([12], 4.2), if the union of the curves $A_{1}, \ldots, A_{k}$ is a minimal resolution of the singular point $\mathrm{p}$, then the Chern divisor of $\mathrm{p}$ is a sum $\sum_{i=1}^{k} a_{i} A_{i}$ where the $a_{i}$ are rational numbers (the usual divisors, as introduced in Chapter I, have always integer coefficients). Thus the Chern divisor of a cusp singularity is equal to the sum of the curves in the resolution, and the Chern divisor of a quotient singularity of type $(3 ; 1,1)$ equals $\frac{1}{3}$ times the only curve in the resolution. The Chern divisor vanishes if only non-singular rational curves with self-intersection number -2 occur in the resolution.

Let $c_{1} \in H^{2}(Y(p), \mathbf{Z})$ be the first Chern class of $Y(p)$. If $K$ is a canonical divisor on $Y(p)$, then its cohomology class under Poincare duality equals $-c_{1}$. For a curve $F_{N}$ we need information on $c_{1}\left[F_{N}\right]=-K F_{N}$. If $F_{N}$ has $\overline{\mathscr{H}} / \Gamma$ as non-singular model, then

$$
c_{1}\left[F_{N}\right]=2 \int_{\mathscr{H} / \Gamma} \omega+\sum_{\nu} C_{1}^{(\nu)} F_{N}
$$

where $\int_{\mathscr{H} / \Gamma} \omega$ is the Euler-Gauß-Bonnet volume of $\mathscr{H} / \Gamma$ and $C_{1}^{(v)}$ runs through the Chern divisors of all the singular points in the compactification of $\mathscr{H}^{2} / \mathrm{G}$ (compare [12], 4.3). Taking into account that $\Gamma=\Gamma_{*}(\mathrm{p})$, that $\left[\mathrm{SL}_{2}(\mathbf{Z}): \Gamma_{0}(\mathrm{p})\right]=\mathrm{p}+1$ and that $\int_{\mathscr{H} \mathrm{SL}_{2}(\mathbf{Z})} \omega=-\frac{1}{6}$ we derive from $(10)$ and Proposition II.5 the following formula for $\mathrm{N}=\mathrm{p}$ :

$$
c_{1}\left[F_{p}\right]=-\frac{\mathrm{p}+1}{6}+\frac{\varepsilon}{3}+2 .
$$

Since $F_{p}$ is non-singular, we have by the adjunction formula

$$
c_{1}\left[F_{p}\right]-F_{p}^{2}=2-2 \pi\left(F_{p}\right)=e\left(F_{p}\right)=2-2 \mathrm{~g}_{*}(\mathrm{p})
$$

where $e\left(F_{p}\right)$ is the Euler number of $F_{p}$. Formulas (11) and (12) imply the following proposition.

Proposition II.6. The self-intersection number of the non-singular curve $F_{p}$ on the Hilbert modular surface $Y(p)$ is given by the formula

$$
F_{p}^{2}=-\left[\frac{\mathrm{p}+1}{6}\right]+2 \mathrm{~g}_{*}(\mathrm{p}) .
$$


From a classical formula for the genus $g_{*}(p)$ it follows that $g_{*}(p)=0$ for $\mathrm{p}=5,13,17,29,41$, that $\mathrm{g}_{*}(\mathrm{p})=1$ for $\mathrm{p}=37,53,61,89,101$, that $g_{*}(p)=2$ for $p=73$, and $g_{*}(p)=3$ for $p=97$. This information covers all the values of $g_{*}(p)$ for $p \leqq 101$. Using suitable estimates, Helling has shown recently ([8]) that for the primes $p$ congruent $1 \bmod 4$ the above are the only values of $\mathrm{p}$ for which $\mathrm{g}_{*}(\mathrm{p})=0$ or $\mathrm{g}_{*}(\mathrm{p})=1$.

Formula (10) implies for the curves $F_{N}(\mathrm{~N}$ not congruent $0 \bmod \mathrm{p})$ the following result $([12], 4.3)$.

Proposition II.7. Let $\mathrm{N}$ be admissible with respect to $\mathrm{p}$ and $\mathrm{N}$ not congruent $0 \bmod \mathrm{p}$. Then for the curve $F_{N}$ on the Hilbert modular surface $Y(p)$ the number $c_{1}\left[F_{N}\right]=-K F_{N}$ can be bounded from below by a number $c_{1}(\mathrm{~N})$ depending only on $\mathrm{N}$ :

$$
c_{1}\left[F_{N}\right] \geqq \mathrm{c}_{1}(\mathrm{~N}) .
$$

For the values of $\mathrm{N}$ occurring below in (13) the curve $F_{N}$ is rational and the corresponding numbers $\mathrm{c}_{1}(\mathrm{~N})$ are as indicated.

$$
\begin{array}{l|rrrrrrrrrrrrrrr}
\mathrm{N} & 1 & 2 & 3 & 4 & 5 & 6 & 7 & 8 & 9 & 10 & 12 & 13 & 16 & 18 & 25 \\
\hline \mathrm{c}_{1} & 1 & 1 & 1 & 1 & 0 & 0 & 0 & 0 & 0 & -2 & -2 & -2 & -2 & -4 & -4
\end{array}
$$

For $\mathrm{N}=1$, the basic configuration shows that $c_{1}\left[F_{1}\right]=1$. Hence $F_{1}$ is an exceptional curve on $Y(p)$.

The curves $F_{N}$ are mapped to themselves by $t$.

Before continuing the study of the curves $F_{N}$ on the Hilbert modular surfaces $Y(p)$, we have to recall the formulas for the arithmetic genus of $Y(p)$ and of $Y(p) / l$ (with the quotient singularities coming from the isolated fixed points of $t$ resolved, see the basic configuration). It was shown in [11] and [12] that

$$
\chi(Y(p))=\frac{1}{4} e\left(\mathscr{H}^{2} / \mathrm{G}\right) .
$$

Formula (1) and the estimate $\zeta_{K}(-1)>\frac{1}{360} \mathrm{p}^{\frac{3}{2}}$ imply:

$$
\chi(Y(p))>\frac{1}{720} \mathrm{p}^{\frac{3}{2}}
$$

and explicit calculations (tables by D. Zagier) show:

$$
\begin{aligned}
& \chi(Y(p))=1 \Leftrightarrow \mathrm{p}=5,13,17 \\
& \chi(Y(p))=2 \Leftrightarrow \mathrm{p}=29,37,41 \\
& \chi(Y(p))=3 \Leftrightarrow \mathrm{p}=53,61,73 .
\end{aligned}
$$

The information contained in the basic configuration and formulas for branched coverings imply

$$
\chi(Y(p) / l)=\frac{1}{2}\left(\chi(Y(p))-\left[\frac{p-29}{24}\right]\right) .
$$


Estimates and explicit calculations give $([11,12])$

$$
\chi(Y(p) / l) \geqq 2 \text { for } \mathrm{p}>317 .
$$

According to (15) the surface $Y(p)$ is not rational for $p>17$.

In the following we assume $p>17$.

Then Propositions I.5, II.4, II.7 together with the adjunction formula imply that $F_{1}, F_{2}, F_{3}, F_{4}$ are exceptional curves. The curves $F_{2}, F_{4}$ exist only if 2 is admissible with respect to $\mathrm{p}$, i.e. if $\delta=1$. The curve $F_{3}$ exists only if 3 is admissible with respect to $p$, i.e. if $\varepsilon=1$.

It was shown in [12] that $F_{3}$ passes through the point $p_{2}$ in the basic configuration, intersecting $B_{2}$ transversally. The curve $F_{2}$ goes through exactly one of the points $p_{3}, p_{4}$, intersecting $L$ transversally. The curve $F_{4}$ passes through $p_{5}$, intersecting $S_{0}$ transversally. In $\mathscr{H}^{2}$ the curve $F_{4}$ can be given by

$$
\begin{aligned}
& z_{1}=t+\frac{1}{2} w_{0} \\
& z_{2}=t+\frac{1}{2} w_{0}^{\prime}
\end{aligned} \quad w_{0}=\frac{\{\sqrt{p}\}+\sqrt{p}}{2} .
$$

In the case that the number 2 is not admissible the Eqs. (18) still determine a curve $F$ on $Y(p)$, which passes through $p_{5}$. We have $l(F)=F$, and it can be shown that $F$ is always exceptional on $Y(p)$. Thus for any $\mathrm{p}$ we have an exceptional curve $F$ which coincides with $F_{4}$ if 2 is admissible.

In the sequel the following terminology will be used. Let $X$ be a complex surface, and $C, D, \ldots$ curves on $X$. If an exceptional curve $E$ on $X$ is blown down, then, in as far as they are different from $E$, we shall speak of the images of $C, D, \ldots C$ a the new si rface as of $C, D, \ldots$. Thus if we say: we blow down $E, F, \ldots$ successively, we mean that first we blow down $E$, then on the new surface the image of $F$, and so on. The basic configuration shows that we can blow down on $Y(p)$ successively the curves $F_{1}, E, B_{1}$. In view of Proposition I.6, the curves $F, F_{2}, F_{3}$ are disjoint and do not intersect any of the curves $F_{1}, E, B$. So we can also blow down the curve $F$; the curve $F_{2}$, then $L$ if $\delta=1$; and the curve $F_{3}$ if $\varepsilon=1$. The non-singular algebraic surface thus obtained will be denoted by $Y^{0}(p)$. It is obtained from $Y(p)$ by blowing down $4+2 \delta+\varepsilon$ times.

We conjecture that (always for $\mathrm{p} \geqq 29) Y^{0}(p)$ is a minimal model, i.e. that it does not contain any exceptional curves (see Chapter III, Remark 1).

Let $c_{1}^{0}$ be the first Chern class of $Y^{0}(p)$ and $K^{0}$ a canonical divisor of $Y^{0}(p)$. For the investigation of the surfaces $Y^{0}(p)$ it is important to know the number $\left(c_{1}^{0}\right)^{2}\left[Y^{0}(p)\right]=\left(K^{0}\right)^{2}$ (see Theorem ROC). This number can be calculated in the following way. Using the second Todd polynomial, we have

$$
\begin{aligned}
\chi(Y(p)) & =\chi\left(Y^{0}(p)\right)=\frac{1}{12}\left(\left(c_{1}^{0}\right)^{2}+e\left(Y^{0}(p)\right)\right) \\
\left(c_{1}^{0}\right)^{2} & =12 \chi(Y(p))-e\left(Y^{0}(p)\right) \\
& =12 \chi(Y(p))-e(Y(p))+4+2 \delta+\varepsilon .
\end{aligned}
$$


By Propositions II.1 and II.2 and the formulas (1), (7), (14) we get for $p \geqq 29$

$$
\left(c_{1}^{0}\right)^{2}\left[Y^{0}(p)\right]=4 \zeta_{\mathrm{K}}(-1)-\frac{\mathrm{h}(-3 \mathrm{p})}{6}-\ell(\mathrm{p})+4+2 \delta+\varepsilon .
$$

It is interesting to observe that $\mathrm{h}(-\mathrm{p})$ drops out. In Table 2 we list for $29 \leqq \mathrm{p} \leqq 317$ the values of $\zeta_{\mathrm{K}}(-1), \mathrm{h}(-\mathrm{p}), \mathrm{h}(-3 \mathrm{p}), \ell(\mathrm{p})$ and $\left(K^{0}\right)^{2}$. It can be proved that $\left(c_{1}^{0}\right)^{2}\left[Y^{0}(p)\right]>0$ for $\mathrm{p} \geqq 89$. This involves estimates concerning $\ell(\mathrm{p})$ which will be carried out in [13]. But we shall be able to prove our classification result in the next Chapter without using this fact. We shall only need $\left(c_{1}^{0}\right)^{2}\left[Y^{0}(p)\right]>0$ for $89 \leqq p \leqq 317$, a fact that follows immediately from Table 2 .

Table 1

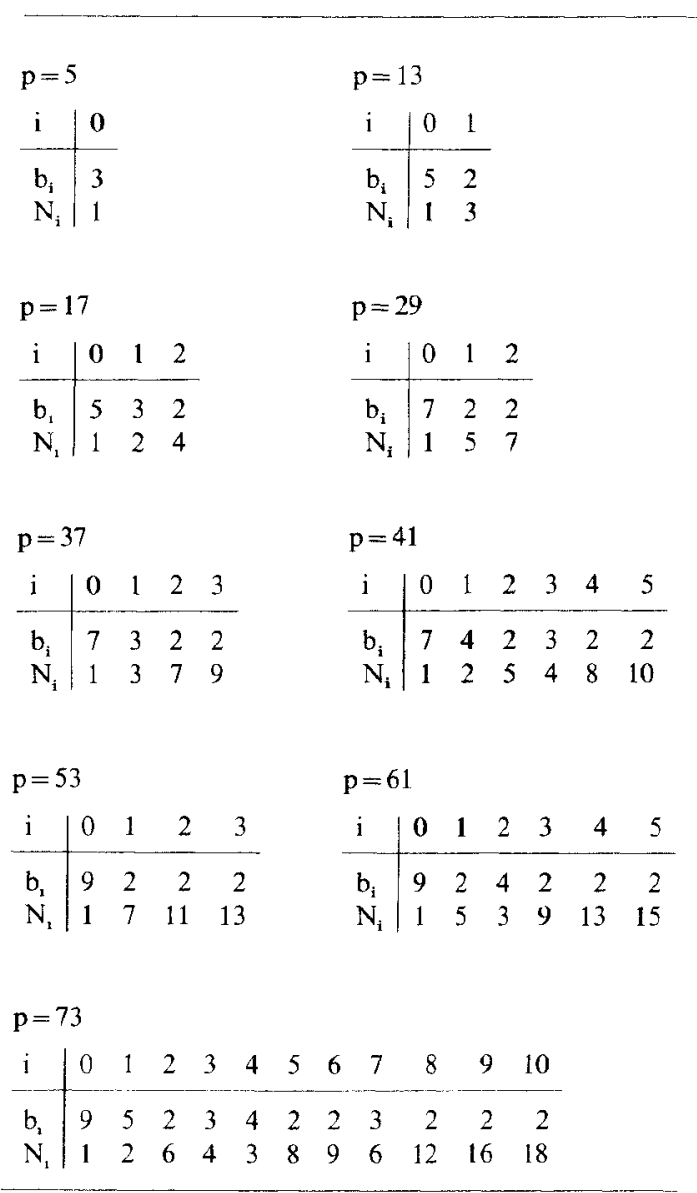


Table 2

\begin{tabular}{rrrrrl}
\hline $\mathrm{p}$ & $6 \zeta_{\mathbf{K}}(-1)$ & $\mathrm{h}(-\mathrm{p})$ & $\mathrm{h}(-3 \mathrm{p})$ & $\ell(\mathrm{p})$ & $\left(c_{1}^{0}\right)^{2}\left[Y^{\mathbf{0}}(p)\right]$ \\
\hline 29 & 3 & 6 & 6 & 5 & 0 \\
37 & 5 & 2 & 8 & 7 & 0 \\
41 & 8 & 8 & 2 & 11 & 0 \\
53 & 7 & 6 & 10 & 7 & 0 \\
61 & 11 & 6 & 8 & 11 & 0 \\
73 & 22 & 4 & 4 & 21 & 0 \\
89 & 26 & 12 & 2 & 21 & 2 \\
97 & 34 & 4 & 4 & 27 & 2 \\
101 & 19 & 14 & 10 & 11 & 4 \\
109 & 27 & 6 & 12 & 17 & 4 \\
113 & 36 & 8 & 6 & 23 & 6 \\
137 & 48 & 8 & 6 & 27 & 10 \\
149 & 35 & 14 & 14 & 15 & 10 \\
157 & 43 & 6 & 16 & 19 & 12 \\
173 & 39 & 14 & 18 & 13 & 14 \\
181 & 57 & 10 & 12 & 25 & 16 \\
193 & 98 & 4 & 8 & 47 & 24 \\
197 & 49 & 10 & 22 & 15 & 18 \\
229 & 81 & 10 & 12 & 29 & 28 \\
233 & 106 & 12 & 10 & 39 & 36 \\
241 & 142 & 12 & 4 & 59 & 42 \\
257 & 120 & 16 & 6 & 39 & 46 \\
269 & 83 & 22 & 14 & 21 & 36 \\
277 & 103 & 6 & 28 & 29 & 40 \\
281 & 150 & 20 & 6 & 49 & 56 \\
293 & 85 & 18 & 22 & 17 & 40 \\
313 & 200 & 8 & 8 & 65 & 74 \\
317 & 101 & 10 & 26 & 21 & 46 \\
\hline & & & & & \\
\hline
\end{tabular}

\section{The Structure of the Surfaces $Y^{\mathbf{0}}(p)$}

In this Chapter we shall prove the main result of this paper, as it is contained in the following theorem.

Theorem III.1. The surfaces $Y(p)$ are

rational surfaces for $\mathrm{p}=5,13,17$;

blown up elliptic $\mathrm{K}_{3}$-surfaces for $\mathrm{p}=29,37,41$;

honestly elliptic surfaces for $\mathrm{p}=53,61,73$;

surfaces of general type for $\mathrm{p} \geqq 89$.

In the course of the proof we shall use the following auxiliary result.

Proposition III.2. Let $X$ be a non-singular algebraic surface with $q(X)=0$ and with $\chi(X) \geqq 3$. Let $\rho: X \rightarrow \mathbf{P}_{1}$ be an elliptic fibring of $X$, and let $C$ be an irreducible curve on $X$ with $\rho(C)=\mathbf{P}_{1}$. If there exists a holo- 
morphic involution $\imath: X \rightarrow X$ without isolated fixed points and leaving $C$ pointwise fixed, then the quotient of $X$ by $\mathrm{l}$ is a rational algebraic surface.

Proof. Since $\chi(X) \geqq 3, X$ is neither a rational surface nor a blown up $\mathrm{K}_{3}$-surface. Therefore $X$ is obtained by blowing up an honestly elliptic surface $Y$ without exceptional curves (Theorem ROC and the remarks thereafter). Let $\sigma: X \rightarrow \mathbf{P}_{1}$ be an elliptic fibring of $X$, induced by an elliptic fibring of $Y$. We shall identify two elliptic fibrings of $X$ if they have set theoretically the same fibres. Then we claim: $\sigma$ is the only elliptic fibring of $X$ (and therefore is equal to the given fibring $\rho$ ). For let $\tau: X \rightarrow \mathbf{P}_{1}$ be another elliptic fibring of $X$ (the base of any elliptic fibring of $X$ is $\mathbf{P}_{1}$ since $q(X)=0$ ). Suppose, there is a fibre $G_{0}$ of $\tau$, which is set theoretically different from all the fibres of $\sigma$. Then $G_{0}$ cannot be contained in a fibre of $\sigma$ because of the theorem of Zariski (loc. cit. in the proof of Proposition I.9). Therefore $\sigma\left(G_{0}\right)=\mathbf{P}_{1}$, hence there is a neighbourhood $U$ of $G_{0}$ on $X$ such that for all the fibres $G$ of $\tau$ contained in $U$, we have $\sigma(G)=\mathbf{P}_{1}$. Since there is only a finite number of reducible fibres of $\tau$, we can find an irreducible fibre $G$ of $\tau$ with $\sigma(G)=\mathbf{P}_{1}$, i.e. $G$ intersects all the components of all fibres of $\sigma$ in a non-negative number of points. Using for the canonical class of $Y$ a formula mentioned in Chapter I, and taking into account that at each blowing up the canonical class of the new surface is the pull back of the canonical class of the original surface plus the exceptional curve obtained, we find that

$$
K_{X}=\sigma^{*}(A)+\sum a_{i} C_{i}, \quad a_{i}>0 .
$$

In this formula $A$ is a divisor class of strictly positive degree on $\mathbf{P}_{1}$ (because $\chi(X) \geqq 3$ ) and the curves $C_{i}$ are components of fibres of $\sigma$. This formula implies that $K_{X} G$ is strictly positive, a contradiction. Hence $\rho$ is the only elliptic fibring of $X$. Since the involution $l$ transforms the fibres of an elliptic fibring of $X$ into the fibres of an elliptic fibring of $X$, we find that $l$ permutes the fibres of $\rho$. Now the curve $C$, left fixed pointwise by $l$, intersects all fibres of $\rho$, hence $t$ transforms every fibre of $\rho$ into itself. Also the restriction of $l$ to a fibre has at least one fixed point. Hence the quotient of any non-singular elliptic fibre of $\rho$ by $z$ is a non-singular rational curve, which has self-intersection 0 on the non-singular quotient surface $Y=X / l$. Since $Y$ is algebraic (for example by [14], Theorem 3.3) and $q(Y)=0$, the proposition now follows from Proposition I.2.

Proof of Theorem III.1. In Chapter II, we have introduced the basic configuration on the surface $Y(p)$.

As was already observed in [11], the information contained in the basic configuration suffices to prove Theorem III. 1 for $\mathrm{p}=5,13$ and 17 . In fact, for $\mathrm{p}=5$, after the blowing down of $F_{1}$ and $E$, the curve $F_{5}$ 
becomes a non-singular rational curve with $F_{5}^{2}=0$ (Propositions I.1 and II.6). Thus Proposition I.2 yields that $Y(5)$ is a rational surface. Similarly, for $\mathrm{p}=13$, after the blowing down of $F_{1}, E$ and $B_{1}$, the curve $F_{13}$ becomes a non-singular rational curve with $F_{13}^{2}=0$, and for $\mathrm{p}=17$ the same holds for the curve $S_{2}$ after the blowing down of $F_{1}, E, B_{1}, F_{17}$ and $C_{1}$ (by Table 1 the self-intersection $S_{2}^{2}=-2$ on $\left.Y(17)\right)$.

From Chapter II we know about the existence and part of the intersection properties of the curves $F_{N}$ - not only for $\mathrm{N}=1$ and $\mathrm{N}=\mathrm{p}$, but for all $\mathrm{N}=1,2,3, \ldots-$ and the curve $F$, which is sometimes identical with $F_{4}$. To prove Theorem III.1 for $p \geqq 29$ we shall use the structure of the configuration formed by the curves of the basic configuration and (some of the curves $F_{N}$ and $F$. From now we assume that $\mathrm{p} \geqq 29$. By the results of Chapter II (see (15)), the arithmetic genus $\chi(Y(p))$ is greater or equal to 2, in particular $Y(p)$ is not a rational surface. Now let $\mathrm{p}=29$. After blowing down $F_{1}, E$ and $B_{1}$ we get $S_{0}^{2}=-1$ and $K S_{0}=+1$. (After the second blowing down we have $B_{1} S_{0}=2$.) We know from Chapter II that $F S_{0}=1$, hence after blowing down $F$ we find $K S_{0}=S_{0}^{2}=0$. Furthermore $F$ does intersect neither $S_{1}$ nor $S_{-1}$. For if $F$ would intersect either one of these curves, $F$ would intersect both of them, since $\imath(F)=F$ and $\iota\left(S_{1}\right)=S_{-1}$. But on $Y(29)$ we have by Table $1: S_{1}^{2}=S_{-1}^{2}=-2$, so if $F$ would intersect $S_{1}$ and $S_{-1}$, after blowing down we would get a violation of Proposition I.6. Hence, if after blowing down $F$ we set $S_{0}=C$ and $S_{1}=D$, then all the assumptions of Proposition I.7 are satisfied and as a consequence $Y(29)$ is a blown up elliptic $\mathrm{K}_{3}$-surface. For $\mathrm{p}=37$ and $\mathrm{p}=41$ the argument is similar. On $Y(37)$ we have $S_{1} F_{3}=S_{-1} F_{3}=1$. Since $S_{1}^{2}=-3$ we find that after blowing down $F_{3}$ we have $S_{1}^{2}=-2$ and can conclude again $S_{1} F=S_{-1} F=0$ on $Y(37)$. After blowing down $F_{1}, E, B_{1}$ and $F$ on $Y(37)$ we can apply again Proposition $I .7$ with $S_{0}=C$ and $S_{1}=D$ to obtain the required result. Similarly, in the case $\mathrm{p}=41$ the curves $F_{1}, E$, $B_{1}, F_{2}, F_{4}(=F)$ and $L$ are blown down, after which Proposition I.7 again yields that $Y(41)$ is a blown up elliptic $\mathrm{K}_{3}$-surface ${ }^{1}$.

\footnotetext{
${ }^{1}$ It can be seen directly that $F$ does not intersect $S_{1}$ and $S_{-1}$ on any surface $Y(p)$. The intersection of $F$ with $S$ can be described as follows (compare [12], $\S 4$ ). If $\overline{\mathscr{H} / \Gamma}$ is the non-singular model for $\boldsymbol{F}$, then $\overline{\mathscr{H}} / \boldsymbol{\Gamma}-\mathscr{H} / \Gamma$ has 3 or 1 points depending on whether 2 is admissible or not. If 2 is not admissible, then the only intersection of $F$ with $S$ is the point $p_{5}$ of the basic configuration. If 2 is admissible, then the three points of intersection are $p_{5}$ and transversal intersections with $S_{w}$ for the two reduced quadratic irrationalities $w$ of the form
}

which always exist.

$$
w=\frac{M+\sqrt{p}}{16}, \quad w \in L(p)
$$

Since $\mathrm{N}_{1}=\frac{\mathrm{b}_{0}^{2}-\mathrm{p}}{4} \neq 4$, the curve $F$ does not intersect $S_{1}$ and $S_{-1}$ (compare Proposition II.5). 
Now we come to the case $p \geqq 53$. Taking into account that for these values of $\mathrm{p}$ the arithmetic genus $\chi(Y(p))$ is greater than 2 , we find from Theorem ROC that $Y(p)$ is either honestly elliptic or a surface of general type. Let $\mathrm{p}=53$. In this case the curve $F_{7}$ intersects $S_{1}$ and $S_{-1}$ (Table 1). Since on $Y(53)$ we have $S_{1}^{2}=S_{-1}^{2}=-2$, it follows from Proposition I.6, I.1, and 1.5 that $F_{7}$ is not an exceptional curve. From Proposition II.7 we know that $K F_{7} \leqq 0$. If $K F_{7}$ would be strictly negative, it would follow from Proposition 1.4 and the adjunction formula that $F_{7}$ is an exceptional curve which is impossible by the remark above. Hence $K F_{7}=0$. Let $Y(53)$ be obtained by blowing up from a surface $Y$ without exceptional curves. Then $F_{7}$ is not blown down to a point, because otherwise the curves $S_{1}$, $S_{-1}$ would again give a contradiction with Proposition I.5 or I.6. It also follows in the same way that none of the curves in the configuration $\left\{S_{1}, F_{7}, S_{-1}, S_{-2}, S_{-3}, S_{3}, S_{2}\right\}$ (compare Table 1) intersects a curve on $Y(53)$ which is blown down to get $Y$. By the adjunction formula $F_{7}^{2}=0$ or $F_{7}^{2}=-2$ on $Y(53)$ and on $Y$. Therefore, $Y$ is not of general type, because all the curves in the configuration have intersection number 0 with $K_{Y}$ and would be mapped to a point under the map belonging to $\left|n K_{Y}\right|$, $\mathrm{n}$ large. But the configuration is connected and not negative definite. This is clear if $F_{7}^{2}=0$. If $F_{7}^{2}=-2$, we have an elliptic ( -2$)$-configuration.

For $\mathrm{p}=61$ and $\mathrm{p}=73$ the argument is similar. In the first case one uses the (-2)-configuration $\left\{S_{3}, F_{9}, S_{-3}, S_{-4}, S_{-5}, S_{5}, S_{4}\right\}$ and in the second case the (-2)-configuration $\left\{S_{5}, F_{8}, S_{-5}, S_{-6}, F_{9}, S_{6}\right\}$ (see Table 1).

Next, let $89 \leqq \mathrm{p} \leqq 317$, and let $Y^{\circ}(p)$ be defined as in Chapter II. Then $\left(c_{1}^{0}\right)^{2}\left[Y^{0}(p)\right] \geqq 1$ for all these values of $\mathrm{p}$ (see Table 2). It follows from Proposition I.1 that $Y^{0}(p)$ cannot be obtained by blowing up a surface $Z(p)$ without exceptional curves for which $\left(c_{1}^{0}\right)^{2}(Z(p)) \geqq 0$. Since $Y(p)$ is not rational we derive from Theorem $\mathrm{ROC}$ that $Y^{0}(p)$ and hence $Y(p)$ is of general type.

In fact, it can be proved that for all $\mathrm{p} \geqq 89$ the inequality $c_{1}^{2}\left(Y^{0}(p)\right) \geqq 1$ holds, but this requires some estimates ([13]). For $p>317$ we therefore apply the following argument. The involution $l$ on $Y(p)$ induces an involution $l^{(0)}$ on $Y^{0}(p)$, which has no isolated fixpoints. $l^{(0)}$ leaves the curve $F_{p}$ pointwise fixed. By Chapter II, formula (15), we have $\chi\left(Y^{0}(p)\right) \geqq 4$, therefore by Theorem ROC $Y^{0}(p)$ is either honestly elliptic or of general type. Now we know from Chapter II that $Y^{0}(p) / l^{(0)}$ is not rational since the arithmetic genus $\chi\left(Y^{0}(p)\right) \geqq 2$. Therefore, by Proposition III.2, if $Y^{0}(p)$ were an honestly elliptic surface, $F_{p}$ would be contained in a fibre of an elliptic fibring of $Y^{0}(p)$, which would be induced by an elliptic fibring of an honestly elliptic surface $Y$ without exceptional curves. Certainly this is not possible in case the genus of $F_{p}$ is at least 2. For then also a fibre of an elliptic fibring of $Y$ would contain a curve of virtual genus at least 2, which is impossible, either by Kodaira's classification 
of fibres in elliptic fibrings, or by the adjunction formula and Zariski's theorem (loc. cit. in Chapter I). Now by Hellings recent result, cited after Proposition II.6, for $\mathrm{p} \geqq 317$ the curve $F_{p}$ is always of genus at least two. Therefore, we can finish our proof by applying Hellings result. However, it is also possible to exclude the remaining cases, i.e. the cases for which the genus of $F_{p}$ is 0 or 1 , in the following way. If $Y^{0}(p)$ would be honestly elliptic, $Y^{0}(p)$ would be obtained from a minimal honestly elliptic surface $Y$ by blowing up. All we have to show is that the image of $F_{p}$ on $Y$ (either curve or point) cannot be included in a fibre of the elliptic fibring of $Y$. Suppose $F_{p}$ is an elliptic curve. Since $\mathrm{h}(-\mathrm{p})$ and $\mathrm{h}(-3 \mathrm{p})$ are strictly positive, the curve $D_{1}$ and the curves $C_{1}, C_{1}^{\prime}$ are always present on $Y(p)$, and none of these curves has been touched by the blowing down from $Y(p)$ to $Y^{0}(p)$. From the formula for $K_{Y}$, given in Chapter I, and the fact that if the non-singular surface $Z$ is obtained from the non-singular surface $X$ by blowing up a point to the curve $E$, then $K_{Z}$ is the inverse image of $K_{X}$ plus $E$, it follows that for every irreducible curve $A$ on $Y^{0}(p)$, not contained in a fibre, we have that $K_{Y^{\circ}(p)} A \geqq 1$. So if on the surface $Y^{0}(p)$ the curve $F_{p}$ would be contained in a fibre, the curves $D_{1}, C_{1}$ and $C_{1}^{\prime}$ would be contained in the same fibre. Furthermore, Kodaira's classification ([16], p. 565) or the adjunction formula combined with Zariski's theorem yield that if $F_{p}$ would be elliptic, and contained in a fibre, this curve would be the only component of the corresponding fibre on $Y$, necessarily nonsingular. In particular, the curve $D_{1}$ would be blown down to a point. Since on $Y^{0}(p)$ the curves $F_{p}$ and $D_{1}$ intersect in two points this would imply that on $Y$ the curve $F_{p}$ would be singular. This contradiction concludes the proof for the case that $F_{p}$ is elliptic. Finally, we come to the case that $F_{p}$ is rational. (The facts used about the structure of fibres in elliptic fibrings follow again from Kodaira's classification or alternatively from the adjunction formula combined with Zariski's theorem). We consider several cases separately. Let us first assume that $F_{p}$ is not blown down to a point on $Y$. Then either there is a fibre on $Y$ consisting of $F_{p}$ only, or not. In the first case this fibre has one singular point, either an ordinary double point or a cusp. The curve $D_{1}$ goes to a point on $Y$, as do $C_{1}$ and $C_{1}^{\prime}$. But this leads to a contradiction: since $D_{1} \cap F_{p}$ consists of two points on $Y^{0}(p)$, the only singularity of $F_{p}$ on $Y$ has to be the image point of $D_{1}$. But then the curves $C_{1}$ and $C_{1}^{\prime}$ can never meet on $F_{p}$. In the second case, $F_{p}$ has to be non-singular on $Y$, and $D_{1}$ therefore has to go to a curve on $Y$. But then the fibre of $Y$, containing $F_{p}$ and $D_{1}$ can only exist of these two curves, necessarily non-singular. Hence $C_{1}$ and $C_{1}^{\prime}$ go to a nonsingular point of $F_{p}$ on $Y$, but then on $Y^{0}(p)$ they cannot meet on $F_{p}$. Finally, suppose $F_{p}$ is blown down to a point on $Y$. Since $F_{p} \cap D_{1}$ consists of two points, $D_{1}$ does not go to a point on $Y$. But then the image of $D_{1}$ is singular and the only component of a fibre on $Y$. Hence also $C_{1}$ and $C_{1}^{\prime}$ 
are blown down to a point on $Y$. This gives the last contradiction, since three curves going down to a point can never meet as $F_{p}, C_{1}$ and $C_{1}^{\prime}$ do on $Y^{0}(p)$.

Remark 1. If we calculate for $29 \leqq \mathrm{p} \leqq 73$ the number $\left(c_{1}^{0}\right)^{2}\left(Y^{0}(p)\right)$, we find it to be 0 . Thus for all these cases $Y^{0}(p)$ is a minimal surface, in accordance with the conjecture stated at the end of Chapter II. In fact, the conjecture can also be proved for some values of $\mathrm{p} \geqq 89$, see [25].

Remark 2. The reader can easily convince himself of the fact that for $\mathrm{p} \leqq 73$ the propositions of Chapter I can be used to give several alternative proofs of the statement of Theorem III.1.

Remark 3. Since all $\mathrm{K}_{3}$-surfaces are homeomorphic $([18]$, Theorem 13) and hence simply connected, and since all rational surfaces are simply connected we have that $Y(p)$ is simply connected for $p=5,13,17,29,37$ and 41. Recently, A. Kas has proved that also the honestly elliptic Hilbert surfaces $Y(53), Y(61)$ and $Y(73)$ are simply connected.

\section{References}

1. Andreotti, A.: On the complex structures of a class of simply connected manifolds. In: Algebraic geometry and Topology. A symposium in honour of S. Lefschetz. Princeton, N.J.: Princeton University Press 1957

2. Blumenthal, O.: Über Modulfunktionen von mehreren Veränderlichen. Math. Ann. 56, 509-548 (1903) and 58, 497-527(1904)

3. Bombieri, E.: The pluricanonical map of a complex surface. In: Several Complex Variables I, Maryland 1970. Lecture Notes in Mathematics 155. Berlin-HeidelbergNew York: Springer 1970

4. Brieskorn, E.: Über die Auflösung gewisser Singularitäten von holomorphen Abbildungen. Math. Ann. 166, 76-102 (1966)

5. Freitag, E.: Über die Struktur der Funktionenkörper zu hyperabelschen Gruppen I, II. Journal f. d. r. u. a. Math. (Crelle) 247, 97-117 (1971) and 254, 1-16 (1972)

6. Hammond, W.F.: The Hilbert modular surface of a real quadratic field. Math. Ann. 200, 25-45 (1973)

7. Hecke, E.: Höhere Modulfunktionen und ihre Anwendungen auf die Zahlentheorie. Math. Ann. 71, 1-37 (1912) (= Mathematische Werke, 21-57)

8. Helling, H.: Note über das Geschlecht gewisser arithmetischer Gruppen. Math. Ann. 205, 173-179 (1973)

9. Hirzebruch, F.: Über vierdimensionale Riemannsche Flächen mehrdeutiger analytischer Funktionen von zwei komplexen Veränderlichen. Math. Ann. 126, 1-22 (1953)

10. Hirzebruch, F.: Topological methods in algebraic geometry. Grundl. Math. Wissensch. Band 131. Third edition. Berlin-Heidelberg-New York: Springer 1966

11. Hirzebruch, F.: The Hilbert modular group and some algebraic surfaces. Intern Symp. Number Theory Moscow 1971. To appear

12. Hirzebruch, F.: Hilbert modular surfaces. L'Enseignement Mathématique 19, 183-281 (1973)

13. Hirzebruch, F., Zagier, D.: The rough classification of Hilbert modular surfaces. In preparation 
14. Kodaira, K.: On Kähler varieties of restricted type. Ann. of Math. 60, 28-48 (1954)

15. Kodaira, K.: On compact complex analytic surfaces I. Ann. of Math. 71, 111-152 (1960)

16. Kodaira, K.: On compact complex analytic surfaces II. Ann. of Math. 77, 563-626 (1963)

17. Kodaira, K.: On compact complex analytic surfaces III. Ann. of Math. 78, 1-40 (1963)

18. Kodaira, K.: On the structure of compact complex analytic surfaces I. Am. J. of Math. 86, 751-798 (1964)

19. Kodaira, K.: On the structure of complex analytic surfaces, IV. Am. J. of Math. 90. 1048-1066 (1968)

20. Mumford, D.: The topology of normal singularities of an algebraic surface and a criterion for simplicity. Publ. Math. de l'Institut des Hautes Études Scientifiques 9 (1961)

21. Prestel, A.: Die elliptischen Fixpunkte der Hilbertschen Modulgruppen. Math. Ann. 177, 181-209 (1968)

22. Prestel, A.: Die Fixpunkte der symmetrischen Hilbertschen Modulgruppe zu einem reell-quadratischen Zahlkörper mit Primzahldiskriminante. Math. Ann. 200, 123-139 (1973)

23. Safarevič, I., et al.: Algebraic surfaces. Proc. Steklov Inst. of Math., Am. Math. Soc., Providence, R. I. (1967)

24. Serre, J.-P.: Le problème des groupes de congruence pour $S L_{2}$. Ann. of Math. 92, 489-527 (1970)

25. Van de Ven, A.: Some remarks on Hilbert modular surfaces of general type. To appear

\section{F. Hirzebruch}

Mathematisches Institut der Universität

Wegelerstraße 10

D-5300 Bonn

Federal Republic of Germany
A. Van de Ven

Mathematisch Instituut

Rijksuniversiteit te Leiden

Wassenaarseweg 80

Leiden/The Netherlands

(Received September 2, 1973) 